\title{
RING-mutant Mdm2-M459I Confers Anti-apoptotic Effect in Primary Cells
}

\section{Takumi Mikawa', Matilde E LLeonart ${ }^{2}$, Akifumi Takaori-Kondo ${ }^{3}$, Masayuki Yokode ${ }^{4}$ and Hiroshi Kondoh ${ }^{1,5 *}$}

${ }^{1}$ Department of Diabetes, Endocrinology and Nutrition, Graduate School of Medicine, Kyoto University, Kyoto, 606-8507, Japan

${ }^{2}$ Department of Pathology, Hospital Vall de'Hebron, Paseo Vall d'Hebron 119-129, 08035, Barcelona, Spain

${ }^{3}$ Department of Hematology/Oncology, Graduate School of Medicine, Kyoto University, Kyoto, 606-8507, Japan

${ }^{4}$ Department of Clinical Innovative Medicine, Translational Research Center, Kyoto University Hospital, Kyoto, 606-8507, Japan

${ }^{5}$ Department of Geriatric Unit, Kyoto University Hospital, Kyoto, 606-8507, Japan

\section{Image Article}

Recently, we reported that a novel RING-mutation, Mdm2-M459I, greatly impairs its ubiquitin ligase activity. Mdm2-M459I stabilizes glycolytic enzyme PGAM, followed by transformation of mouse embryonic fibrobalsts (MEFs) [1]. Here we addressed a question whether Mdm2-M459I could also affect anti-apoptotic effect, the other hallmark of cancer. We compared three mutants of Mdm2 described in COSMIC database; $\mathrm{Y} 281 \mathrm{H}$ and $\mathrm{W} 329 \mathrm{G}$ are located in its central
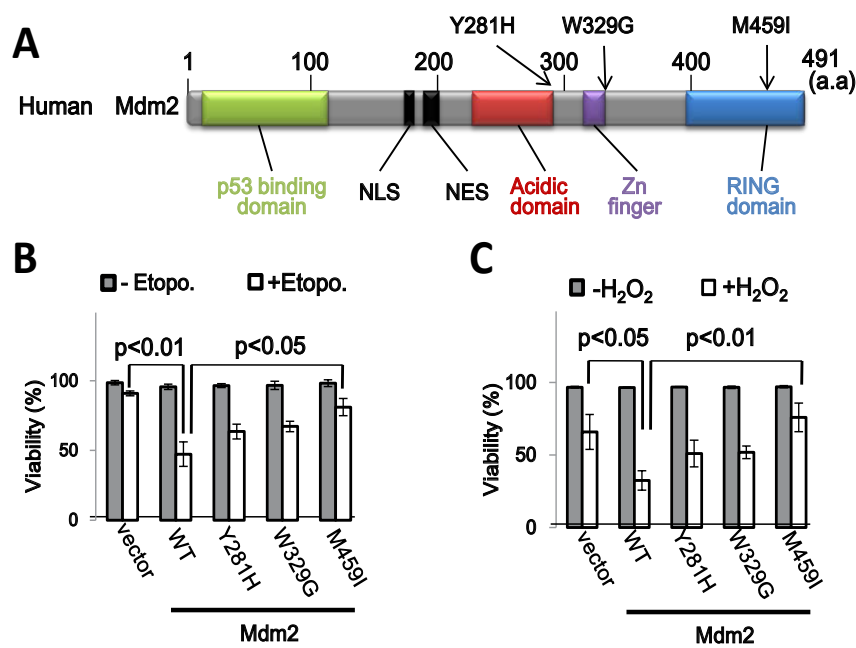

Figure 1: (A) Schematic diagram of human Mdm2 and the location of cance related mutations in Mdm2 according to COSMIC (Catalogue of Somatic Mutations in Cancer) database (http://www.sanger.ac.uk/genetics/CGP cosmic/). (B and C) Primary MEFs were transfected with various Mdm2 expression vectors or empty vector. Cell viability after exposure to $50 \mu \mathrm{M}$ Etoposide for 24 hours (B) or exposure to $50 \mu \mathrm{M} \mathrm{H} \mathrm{H}_{2}$ for 24 hours (C) as measured by tripan blue stain .Error bar is indicate $\mathrm{SE}$.
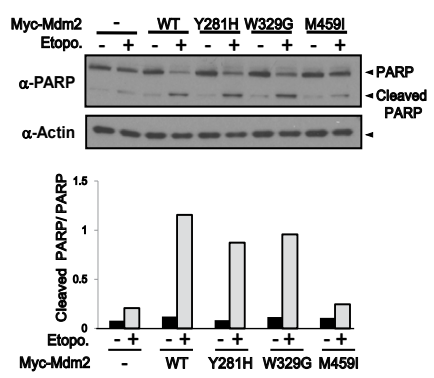
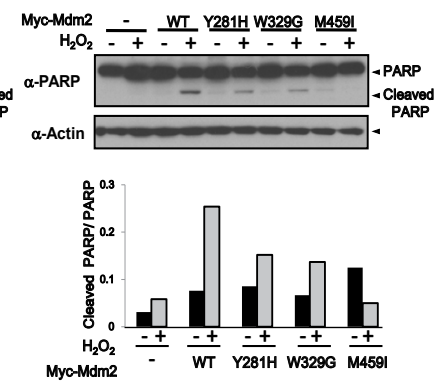

Figure 2: ( $A$ and $B$ ) Various mutant or wild type Mdm2 expressing MEFs were exposed to Etoposide (A) or $\mathrm{H}_{2} \mathrm{O}_{2}(B)$. Apoptotic marker (cleaved PARP) were detected by immunoblotting using anti-PARP antibody (Top), and band intensities were used to assess the level of Cleaved PARP relative to those of full length PARP in each sample (Bottom). domain, while M459I in the RING finger motif (Figure 1A). Primary MEFs transfected with wild type or mutant versions of Mdm2 were exposed to oxidative stress or DNA damage. Noteworthy, only M459I mutation restored the viability of primary MEFs in these conditions (Figure 1B), associated with decrease of cleaved PARP (Figure 2A and B). Thus Mdm2-M459I also plays anti-apoptotic role as oncogene.

\section{Acknowledgement}

This work was supported in part by grants from the Global COE program "Center for Frontier Medicine," from the Japan Society for the Promotion of Science, from the Ministry of Education, Culture, Sports, Science, and Technology of Japan, from Japan Science and Technology Agency, and by JST, CREST.

\section{Reference}

1. Mikawa T, Maruyama T, Okamoto K, Nakagama H, Lleonart ME, et al. (2014) Senescence-inducing stress promotes proteolysis of glycolytic enzyme phosphoglycerate mutase via ubiquitin ligase Mdm2. J Cell Biol 204: 729-745.
*Corresponding author: Hiroshi Kondoh, Department of Diabetes, Endocrinology and Nutrition, Graduate School of Medicine, Kyoto University, Kyoto, 606-8507, Japan, Tel: 0081(0) 75-751-3465; Fax: 0081(0) 75-771-9784; E-mail: hkondoh@kuhp.kyoto-u.ac.jp

Received September 29, 2014; Accepted September 29, 2014; Published October 03, 2014

Citation: Mikawa T, LLeonart ME, Takaori-Kondo A, Yokode M, Kondoh H (2014) RING-mutant Mdm2-M459I Confers Anti-apoptotic Effect in Primary Cells. J Cyto Histol 5: i103. doi:10.4172/2157-7099.1000i103

Copyright: @ 2014 Mikawa T, et al. This is an open-access article distributed under the terms of the Creative Commons Attribution License, which permits unrestricted use, distribution, and reproduction in any medium, provided the original author and source are credited. 